\title{
Fluoride and dental caries
}

\author{
George E Camilleri
}

Correspondence: $\quad$ Prof. G.E Camilleri, Department of Dental Surgery, Medical School, Gwardamangia MSD 08.

Fluorine was probably discovered by the chemist Scheele in 1771 and eventually isolated in 1886 by Moissan. Its presence in bones and teeth was initially disputed but eventually confirmed in the middle of the eighteenth century. Berzelius (1822) suspected its presence in water and in 1846 Wilson reported that the water supply to a brewery in Edinburgh contained Calcium fluoride. Although a correlation between fluoride and dental caries was suspected at the turn of the century and some anti-caries preparations were then available commercially this was not followed up till later. An American dental surgeon, Frederick McKay noted in 1901, that many of his patients were suffering from a peculiar staining of the teeth (mottling) known to the local inhabitants as "Colorado stain". This was the start of an exciting and painstaking series of epidemiological studies which lead to, what many consider, one of the major public health measures in preventive medicine. Mckay laboured for over 30 years linking the mottling directly to the water supply and eventually to a high water fluoride content. These classic epidemiological studies not only recognised the cause for the mottling but also highlighted the possible caries preventive value of the fluoride in water ${ }^{1}$. The US Public Health Service appointed another public health dentist, $\mathrm{H}$ Trendley Dean to continue McKay's work. His conclusions were that near maximal reduction in caries experience occurred with a concentration of $1 \mathrm{ppm} F$ in the drinking water and only the very mildest form of mottled enamel may develop in about 10 per cent of the group. Thus at a fluoride level of $1 \mathrm{ppm}$ the risk of mottling was negligible whilst the caries preventive action is at about its most effective level. Ainsworth, in the UK, applying similar epidemiological methods, confirmed in 1933, that the endemic concentration of tooth mottling in Maldon, England was directly related to the fluoride level in the water supply. These and other surveys confirmed the relationship between fluoride concentration in drinking water, mottled enamel and the incidence of dental caries. Enough evidence was available to recommend the adjustment of the fluoride level in $\mathrm{F}$ deficient water supplies as a dental caries preventive measure. There are many natural water sources which have a fluoride level around the optimal 1 ppm with resultant benefit to the dental health of the persons who have a lifelong experience of drinking this water. When the fluoride level is low, the health authorities in many countries have adjusted their water supplies with a dramatic improvement in the caries level. Water sources with an excessive fluoride content have been adjusted to lower the fluoride content to an optimal level with elimination of the mottling and retention of the anti-caries activity. Unfortunately, the addition of fluoride to water with a sub-optimal content ran into severe opposition. Sodium fluoride in large doses is used as a rat poison and some fringe 'pseudo-scientific' societies put up strong anti-fluoridation campaigns, citing many health scares. In some instances these actions succeeded in blocking or even reversing water fluoridation schemes. These controversies spurred many Health Authorities, and National and International Bodies instigated scientific investigations in many parts of the world which confirmed the safety of the measure. Although court judgements, the WHO and the FDI (Federation Dentaire Internationale) pronounced in favour of the health measure the impetus of water fluoridation has been slowed down.

Several mechanisms have been proposed to explain the mode of action of the fluoride ion in preventing dental caries and it is probable that it acts in a number of ways. The hypothesis that has received most attention is that the fluoride forms a fluorapatite crystal which is less acid soluble and more resistant to the demineralising action of the acids formed by the plaque bacteria. The fluoride must be available systemically during tooth development which occurs in utero and during the early years of childhood. However it is now accepted, that in the erupted tooth there is a continual exchange of minerals at the interface between surface enamel and the oral environment. Surface enamel, which contains a much higher fluoride concentration than the rest of the enamel, is not the stable tissue originally believed but there is a continual activity of demineralisation and remineralisation taking place. The presence of fluoride topically favours the precipitation of crystals of fluorapatite which are more resistant to demineralisation. This mechanism is only possible prior to the breakdown of the supporting organic matrix and cavity formation. Both systemic and topical administration of the fluroide may be utilised to form the more caries resistant fluorapatite crystal. Another possible mode of action is that fluoride is a well known enzyme inhibitor and its presence in dental plaque reduces bacterial acid production. There might also be an effect on the morphology of teeth, with the formation of shallower fissures and less steep cusps thus making the tooth less susceptible to dental caries. These mechanisms are not mutually exclusive and fluoride acts in a multi-factorial way in reducing dental caries, both through systemic intake and by topical application or release. Their relative importance varies and different mode of presentation of the fluoride may act in different ways. Fluoride is effective, though to varying degrees, both when given during tooth formation (pre-eruptive) and 
after tooth eruption. It is of benefit even in the elderly and there is much scope in utilising fluoride preparations to prevent cervical or root caries.

The concentration and availability of fluoride in foods is rather low, the only two common dietary constituents high in fluoride are fish (skin and bones) and tea. The fluoridation of water supplies is the most effective method, has the highest cost benefit ratio and is not dependent on individual co-operation. There may however be logistic, financial or cultural reasons preventing its use. Other ways of utilising fluoride as an anticaries measure are utilised. Schemes have been devised to add fluoride to school water supplies only, or added to salt, milk or fruit juice. Fluoride tablets, drops or mouth rinses are readily available but require the cooperation of the child and parents for regular administration. Professionally applied fluorides by members of the dental health team often utilising higher concentrations, in the form of fluoride solutions or varnishes give good results. A recent development is the incorporation of fluoride in tooth filling materials with a theoretical slow release at the tooth/filling interface and thus reduction of risk of recurrent caries. The commonest method applied universally is the addition of some form of fluoride preparation to toothpaste. Nowadays most toothpaste preparations contain fluoride and this single measure is said to account for the dramatic fall in the incidence of dental caries in many industrialised countries.

There have been many disorders attributed to the toxicity of fluoride and the Royal College of Physicians of England in $1976^{2}$ appointed a commission to study the problem. It drew up a list of disorders which häve been claimed to be caused or aggravated by fluoridation. The effect of water fluoridation on general health has been thoroughly investigated in population studies and no evidence has been found that the consumption of water containing approximately $1 \mathrm{ppm} \mathrm{F}$ is associated with any harmful effect.

The greater availability and use of fluoride containing products has been blamed for the slight increase reported in some studies of minor blemishes in the dental enamel.

\section{The Malta scene}

The caries level in Malta is said to be low although the problem is certainly not negligible and there are some indications that it may be rising 3.4 .

The underground water which until recently was the only source of water had a varied fluoride content depending on the aquifer but all sources contained at least $0.55 \mathrm{ppm}$ (with an optimal $1 \mathrm{ppm}$ in Gozo) and some having a higher than optimal level with the resultant mottling of the teeth. In Malta, we have a complex water supply system which utilises various sources and makes water fluoridation complex and relatively expensive. The introduction of reverse osmosis has drastically lowered the fluoride content in the water as the naturally occurring fluoride in sea water is lost in the osmosis process ${ }^{5}$.The great increase in the use of bottled mineralised drinking water has also lessened the amount of available fluoride although some bottled water manufacturers are trying to remedy the situation. In Malta, as distinct from Gozo, the fluoride content of drinking water has been reduced by about $70 \%$ which is unfortunate and may have a deleterious effect on dental health.

A survey of fluorosis in Malta using the modified Dean's community index of dental fluorosis gave a complex picture with Gozo having $23.8 \%$ of questionable fluorosis (Malta 3.4\%) whilst in the Mild to Severe categories Malta had higher levels ${ }^{6}$. These discrepancies are difficult to explain but one must remember that there are several other conditions, including some hereditary developmental enamel disorders which cause non-fluoride opacities and distinguishing between fluoride and non-fluoride opacities can be very difficult especially under survey conditions.

The present viewpoint is that, in Malta, fluoridation of public water supplies is not warranted ${ }^{7}$. The regular use of fluoridated toothpaste is highly recommended and other fluoride measures, such as fluoride mouth rinses, fluoride tablets or professional topical application utilised when indicated as in children with rampant caries or undergoing orthodontic treatment. The use of topical fluoridation in the elderly person to prevent cervical root caries is an underused preventive measure which should be encouraged. Detailed data for caries incidence, fluoride levels in drinking waters and dental mottling are required to fine tune the policy on the use of fluoride as a public health and/or clinical tool in dental caries prevention. Surveys at regular intervals are required to monitor results and check the efficacy of preventive measures. Finally, it may be salutary to emphasise that although fluoride is an important dental caries preventive measure, it is essential to control the intake of refined sugar, fruit juices and soft drinks.

\section{References}

1. Murray JJ, AJ Rugg-Gunn \& GN Jenkins: Fluorides in Caries Prevention. Wright 1991. 3rd ed.

2. Royal College of Physicians. (1976) Fluoride Teeth and Health. Royal College of Physicians London.

3. Mangion JJ \& C Olivieri Munroe: Preliminary Studies on Oral Health Conditions in Malta. St Luke's Hosp. Gaz. 1968 3:112-115.

4. Galea H: The Dental Health of seven year old children in Malta state run schools. St Luke's Hosp. Gaz. 1971; 6:125-133.

5. Vella AJ \& Borg V. Changes in Fluoride content of Maltese Tap Waters: Implications for Oral Health. Maltese Med J 1989; 1(3):14-17.

6. Vassallo P: M.Sc. Thesis University of London. 1997

7. Galea $\mathrm{H}$; A strategy for children at high risk of developing dental caries. Smile for Health. 1997; pp3-10. 
The copyright of this article belongs to the Editorial Board of the Malta Medical Journal. The Malta Medical Journal's rights in respect of this work are as defined by the Copyright Act (Chapter 415) of the Laws of Malta or as modified by any successive legislation.

Users may access this full-text article and can make use of the information contained in accordance with the Copyright Act provided that the author must be properly acknowledged. Further distribution or reproduction in any format is prohibited without the prior permission of the copyright holder.

This article has been reproduced with the authorization of the editor of the Malta Medical Journal (Ref. No 000001) 